Real Analysis Exchange

Vol. 24(2), 1998/99, pp. 607-614

Kandasamy Muthuvel, University of Wisconsin, Department of Mathematics, 800 Algoma Blvd., Oshkosh, WI 54901. e-mail: muthuvel@uwosh.edu

Arnold W. Miller, University of Wisconsin, Department of Mathematics, Van Vleck Hall, 480 Lincoln Drive, Madison, WI 53706. e-mail:

miller@math.wisc.edu

\title{
EVERYWHERE OF SECOND CATEGORY SETS
}

\begin{abstract}
The main result of this paper states the following. For each natural number i, let $G_{i}$ be a proper additive subgroup of the reals, $A_{i}$ a set that contains no arithmetic progression of length three, $H_{i}$ a basis for the vector space $\mathbb{R}$ over the field of rationals, and $E^{+}\left(H_{i}\right)$ the set of all finite linear combinations from the elements of $H_{i}$ with nonnegative rational coefficients. Then the complement of a finite union of sets $G_{i} \cup A_{i} \cup E^{+}\left(H_{i}\right)$ is everywhere of second category. We also prove that the complement of a union of fewer than continuum many translates of sets that have distinct distances is everywhere of second category.
\end{abstract}

\section{Introduction}

P. Erdös and S. Kakutani [3] showed that the continuum hypothesis is equivalent to the statement that the set $\mathbb{R}$ of real numbers can be partitioned into countably many pieces such that each piece has distinct distances. (That is, if $x, y, z, w$ are real numbers with $|x-y|=|z-w|$, then $\{x, y\}=\{z, w\}$.) Under the assumption of the continuum hypothesis, K. Kunen [6] generalized that, for each positive integer $n, \mathbb{R}^{n}$ can be partitioned into countably many pieces such that each piece has distinct distances. It is interesting to note that, by using the fact that the set $\mathbb{R}$ can be partitioned into countably many sets such that each set contains no arithmetic progression of length three [1, Thm 1.1], K. Ciesielski [2, Thm 3] showed that there exists a uniformly anti-Schwartz function from the reals $\mathbb{R}$ to the natural numbers $\mathbb{N}$.

Key Words: everywhere of second category, arithmetic progression, set having distinct distances

Mathematical Reviews subject classification: 26A03, 26A15, 04A15, 04A20

Received by the editors May 28, 1998 
It is natural to look at the thickness of the complement of a finite union of the above mentioned sets. Suppose $H$ is a basis for the vector space $\mathbb{R}$ over the field of rationals, $P$ is a subset of $\mathbb{R}$ that has distinct distances, $A$ is a subset of $\mathbb{R}$ that has no arithmetic progression of length three, $G$ is a proper additive subgroup of $\mathbb{R}$, and $E^{+}(H)$ is the set of all finite linear combinations from the elements of $H$ with nonnegative rational coefficients. Note that any $H$ set is a $P$ set and any $P$ set is an $A$ set.

In this paper we prove that the complement of a finite union of sets of the form $A \cup G \cup E^{+}(H)$ is everywhere of second category. This is a generalization of a classical result of Sierpiński "the complement of a Hamel basis is everywhere of second category" and a result in [9] "the complement of a finite union of Hamel bases is everywhere of second category." We also prove that the complement of a union of fewer than continuum many translates of sets of the form $P+G$ is everywhere of second category and this is a generalization of Theorem 7 in [7] that the complement of a union of fewer than continuum many translates of Hamel bases is everywhere of second category.

It is interesting to note that there is an additive subgroup, namely, the group generated by the set $H$, which is everywhere of second category. The proof of this follows from the proof of Theorem 1 in [4] with minor suitable modification. Under the assumption of the continuum hypothesis, $E^{+}(H)$ is a Lusin set for some set $H$ (see [4]). Under the assumption of Martin's axiom, $E^{+}(H)$ is a meager set for some set $H$ (see [8]).

\section{Notation}

$\mathbb{R}$ denotes the set of all real numbers, $\mathbb{N}$ is the set of all natural numbers and $\mathbb{Q}$ is the set of all rational numbers. Let $A$ and $B$ be subsets of $\mathbb{R}$. The symbols $A-B$ and $A B$ stand for the sets $\{x-y: x \in A$ and $y \in B\}$ and $\{x y: x \in A$ and $y \in B\}$, respectively. For $r \in \mathbb{R}, A+r=\{x+r: x \in A\}$. The notation $A \backslash B$ stands for the set-theoretic difference of sets $A$ and $B$. All sets considered in this paper are subsets of reals.

\section{$3 \quad$ Results}

Theorem 1. For each $i \in \mathbb{N}$, let $G_{i}$ be a proper additive subgroup of the reals, $A_{i}$ a set that contains no arithmetic progression of length three, $H_{i}$ a basis for the vector space $\mathbb{R}$ over the field of rationals, and $E^{+}\left(H_{i}\right)$ the set of all finite linear combinations from the elements of $H_{i}$ with nonnegative rational coefficients. Then the complement of a finite union of sets $G_{i} \cup A_{i} \cup E^{+}\left(H_{i}\right)$ is everywhere of second category. 
First, we prove the following lemmas.

Lemma 1. Let I be a nonempty open interval and let $F$ be a meager subset of $\mathbb{R}$. Then the set $\mathbb{N}(I \backslash F)$ contains a translated copy of every countable bounded subset of $\mathbb{R}$.

Proof. Let $B$ be a countable bounded subset of $\mathbb{R}$. Then for some nonempty open interval $J, J+B \subseteq m I$ for sufficiently large $m \in \mathbb{N}$. Since $\mathbb{N} F-B$ is meager, $J$ is not contained in the set $\mathbb{N} F-B$. Let $j$ be an element of $J \backslash(\mathbb{N} F-B)$. Then $j+B \subseteq(\mathbb{N} I) \backslash(\mathbb{N} F) \subseteq \mathbb{N}(I \backslash F)$.

Lemma 2. Let $\kappa$ be an infinite cardinal number smaller than the cardinality of the continuum and let $B$ be a subset of $\mathbb{R}$ of size $\kappa$. If a subset $C$ of $\mathbb{R}$ contains a translated copy of every bounded subset of $\mathbb{R}$ of size $\kappa$, then so does the set $C \backslash\left(E^{+}(H)+B\right)$. (Note that $E^{+}(H)$ is defined in Theorem 1.)

Proof. Assume that the conclusion of the lemma is false. Then, for some bounded subset $D$ of $\mathbb{R}$ of size $\kappa$, the set $(D+r) \cap\left(E^{+}(H)+B\right)$ is nonempty for every $r$ in $\{t \in \mathbb{R}: D+t \subseteq C\}$. For simplicity denote $\{t \in \mathbb{R}: D+t \subseteq C\}$ by $\operatorname{Tr}(D, C)$.

(1) Hence $\operatorname{Tr}(D, C) \subseteq E^{+}(H)+B-D$.

Each nonzero real number can be written uniquely as a finite linear combination of elements of $H$ with nonzero rational coefficients. For each nonzero $t \in B-D$, let $\operatorname{supp}(t)=\left\{h \in H: q_{h} \neq 0\right\}$, where $t=\sum_{h \in H} q_{h} h$. Let $X$ be a bounded subset of $-\left(H \backslash \bigcup_{t \in B-D} \operatorname{supp}(t)\right)$ of size $\kappa$. Since $|D+X|=\kappa$, by the definition of $C$, we have $D+X+r \subseteq C$ for some $r$ in $\mathbb{R}$ and hence $X+r \subseteq \operatorname{Tr}(D, C)$. (In fact $\operatorname{Tr}(D, C)$ contains a translated copy of every bounded subset of $\mathbb{R}$ of size $\kappa$.) According to (1),

(2) $X+r \subseteq E^{+}(H)+B-D$ for some $r$ in $\mathbb{R}$.

Since $\operatorname{supp}(r)$ is finite and $X$ is an infinite subset of $-H$, there exists an element $h_{1}$ in $H$ such that $-h_{1} \in X$ and $h_{1} \notin \operatorname{supp}(r)$. It follows from the definition of $X$ that $h_{1} \notin \operatorname{supp}(t)$ for all $t \in(B-D)$. By (2),

$$
-h_{1}+r \in E^{+}(H)+B-D,
$$

which is impossible, because every element of $E^{+}(H)$ is a finite linear combination from the elements of $H$ with nonnegative rational coefficients and $h_{1} \notin \operatorname{supp}(r) \cup \bigcup_{t \in(B-D)} \operatorname{supp}(t)$. 
Lemma 3. If a subset $C$ of $\mathbb{R}$ contains a translated copy of every finite subset of $\mathbb{R}$, so does $C \backslash G$, where $G$ is any proper additive subgroup of $\mathbb{R}$.

Proof. Let $X$ be a finite subset of $\mathbb{R}$ and let

$$
\operatorname{Tr}(X, C)=\{r \in \mathbb{R}: X+r \subseteq C\} .
$$

It is easy to see that $\operatorname{Tr}(X, C)-\operatorname{Tr}(X, C)=\mathbb{R}$. If $(X+r) \cap G$ is nonempty for every element $r \in \operatorname{Tr}(X, C)$, then

$$
\mathbb{R}=\operatorname{Tr}(X, C)-\operatorname{Tr}(X, C) \subseteq(G-X)-(G-X)=G-X+X,
$$

which contradicts the fact that the index of any proper additive subgroup of $\mathbb{R}$ is infinite. (To see this fact, assume that $\mathbb{R}=G+M$, where $G$ is a proper subgroup of $\mathbb{R}$ and $M$ is a finite subset of $\mathbb{R}$. Then for each $m \in M, \exists n_{m} \in \mathbb{N}$ such that $n_{m} m \in G$. Let $n$ be the least common multiple of the integers $n_{m}$. Then $\mathbb{R}=n \mathbb{R}=n G+n M \subseteq G+n M=G$, which is a contradiction.) Thus $X+t \subseteq C \backslash G$ for some $t$ in $\operatorname{Tr}(X, C)$.

Lemma 4. Let I be a nonempty open interval and let $F$ be a meager subset of $\mathbb{R}$. Given a positive integer $n$, let $M$ be a finite subset of $\mathbb{N}$ so that if $M$ is partitioned into $n$ classes, then at least one class contains an arithmetic progression of length three. (Existence of such a set $M$ follows from Van der Waerden's Theorem, see [5, p.28].) Then there exists a nonzero rational number $d$ and a nonempty open interval $J$ such that $d M+j \subseteq I \backslash F$ for all $j$ in $J$ except for a meager subset of $J$.

Proof. Let $a$ and $b$ be two distinct elements of $I$. Then there exist a nonzero rational number $d$ and a real number $r$ such that $d M+r \subseteq\left(\frac{a}{2}, \frac{b}{2}\right)$. Let $F_{1}=\left(\frac{a}{2}, \frac{b}{2}\right) \cap(F-d M-r)$. Then $F_{1}$ is meager and, $\forall x \in\left(\frac{a}{2}, \frac{b}{2}\right) \backslash F_{1}$, we have $d M+r+x \subseteq I \backslash F$. Consequently, if we let $J=\left(\frac{a}{2}, \frac{b}{2}\right)+r$, then $d M+j \subseteq I \backslash F$ for all $j$ in $\bar{J}$ except for a meager subset of $J$.

Lemma 5. For some $j$ in $J$, we have $(d M+j) \subseteq(I \backslash F)$ and

$$
(d M+j) \cap \bigcup_{i=1}^{n}\left(G_{i} \cup E^{+}\left(H_{i}\right)\right)=\emptyset
$$

where $d, M, F, I, J$ are defined in Lemma 4 and $G_{i}, E^{+}\left(H_{i}\right)$ are defined in Theorem 1.

Proof. Assume that the conclusion of the lemma is false. Then, according to Lemma $4,(d M+j) \cap \bigcup_{i=1}^{n}\left(G_{i} \cup E^{+}\left(H_{i}\right)\right)$ is nonempty for all $j$ in $J$ except 
for a meager subset of $J$. This implies that, for some meager set $F_{2}$,

$$
J \backslash F_{2} \subseteq \bigcup_{i=1}^{n}\left(G_{i} \cup E^{+}\left(H_{i}\right)\right)-d M .
$$

Consequently, because $\mathbb{N}\left(G_{i} \cup E^{+}\left(H_{i}\right)\right) \subseteq\left(G_{i} \cup E^{+}\left(H_{i}\right)\right)$, we have

$$
\mathbb{N}\left(J \backslash F_{2}\right) \subseteq \bigcup_{i=1}^{n}\left(G_{i} \cup E^{+}\left(H_{i}\right)\right)-\mathbb{N} d M .
$$

According to Lemma $1, \mathbb{N}\left(J \backslash F_{2}\right)$ contains a translated copy of every countable bounded subset of $\mathbb{R}$. By applying Lemma(2) $n$ times, we obtain that the set

$$
\mathbb{N}\left(J \backslash F_{2}\right) \backslash \bigcup_{i=1}^{n}\left(E^{+}\left(H_{i}\right)-\mathbb{N} d M\right)
$$

contains a translated copy of every countable bounded subset of $\mathbb{R}$. Note that for any proper additive subgroup $G$ of $\mathbb{R}$, the set $G-\mathbb{N} d M$ is contained in a proper additive subgroup of $\mathbb{R}$. For, since $M$ is a subset of $\mathbb{N}$, the set $G-\mathbb{N} d M$ is contained in the subgroup $G+Z d$. If $G+Z d=\mathbb{R}$, since $d$ is rational, $G+Z=\mathbb{R}$, which is impossible. (For, if $\mathbb{R}=G+Z$, then $\frac{1}{2}=g+x$ for some $g \in G$ and $x \in Z$. Consequently, $0 \neq 2 x-1 \in G$. This implies that the index of $G$ is finite, which is a contradiction.) Now by applying Lemma 3 to $\mathbb{N}\left(J \backslash F_{2}\right) \backslash \bigcup_{i=1}^{n}\left(E^{+}\left(H_{i}\right)-\mathbb{N} d M\right)$, we obtain that the set

$$
\left(\mathbb{N}\left(J \backslash F_{2}\right) \backslash \bigcup_{i=1}^{n}\left(E^{+}\left(H_{i}\right)-\mathbb{N} d M\right)\right) \backslash \bigcup_{i=1}^{n}\left(G_{i}-\mathbb{N} d M\right)
$$

contains a translated copy of every finite subset of $\mathbb{R}$, but

$$
\begin{gathered}
\left(\mathbb{N}\left(J \backslash F_{2}\right) \backslash \bigcup_{i=1}^{n}\left(E^{+}\left(H_{i}\right)-\mathbb{N} d M\right)\right) \backslash \bigcup_{i=1}^{n}\left(G_{i}-\mathbb{N} d M\right)= \\
\mathbb{N}\left(J \backslash F_{2}\right) \backslash \bigcup_{i=1}^{n}\left(\left(G_{i} \cup E^{+}\left(H_{i}\right)\right)-\mathbb{N} d M\right)
\end{gathered}
$$

is an empty set. This contradiction completes the proof of the lemma.

To conclude the proof of the theorem, suppose, to the contrary, that $\mathbb{R} \backslash \bigcup_{i=1}^{n}\left(G_{i} \cup A_{i} \cup E^{+}\left(H_{i}\right)\right)$ is meager in a nonempty open interval $I$. Then, for 
some meager set $F$, the set $I \backslash F$ is contained in the set $\bigcup_{i=1}^{n}\left(G_{i} \cup A_{i} \cup E^{+}\left(H_{i}\right)\right)$. Consequently, $(I \backslash F) \backslash \bigcup_{i=1}^{n}\left(G_{i} \cup E^{+}\left(H_{i}\right)\right) \subseteq \bigcup_{i=1}^{n} A_{i}$. Now according to Lemma 5, $d M+j \subseteq \bigcup_{i=1}^{n} A_{i}$ for some real numbers $d, j$ and $d \neq 0$. Hence

$$
M \subseteq \frac{1}{d}\left(\bigcup_{i=1}^{n} A_{i}-j\right) .
$$

By the definition of $M$ (see Lemma 4), for some $i$, the set $\frac{1}{d}\left(A_{i}-j\right)$ contains an arithmetic progression of length three, which is impossible by the definition of $A_{i}$.

Theorem 2. Let $G$ be a subset of $\mathbb{R}$ with cardinal smaller than the cardinality of the continuum and let $P_{1}, \ldots, P_{n}$ be subsets of $\mathbb{R}$ that have distinct distances. (That is, if $\{x, y, z, w\} \subseteq P_{n}$ and $|x-y|=|z-w|$, then $\{x, y\}=\{z, w\}$.) Then $\left(P_{1}+G\right) \cup \ldots \cup\left(P_{n}+G\right)$ cannot be residual in an interval.

First, we prove the following lemma.

Lemma 6. Let $\kappa$ be an infinite cardinal number smaller than the cardinality of the continuum and let $G$ be a subset of the reals such that $|G|<|\mathbb{R}|$. If $P$ is a set that has distinct distances and $C$ is a set that contains a translated copy of every set of size $\kappa$, so does $C \backslash(P+G)$.

Proof. Assume that the conclusion of the lemma is false. Then, for some subset $X$ of $\mathbb{R}$ of size $\kappa$, the set $(X+r) \cap(P+G)$ is nonempty for every $r \in\{t \in \mathbb{R}: X+t \subseteq C\}$. Denote the set $\{t \in \mathbb{R}: X+t \subseteq C\}$ by $\operatorname{Tr}(X, C)$ and the set $G-X$ by $Y$. Then $\operatorname{Tr}(X, C) \subseteq P+G-X \subseteq P+Y$. Let $a \in \mathbb{R} \backslash Q(Y-Y)$ and let $B=\{t \in \mathbb{R}:\{a,-a\}+t \subseteq \operatorname{Tr}(X, C)\}$. Then $\{a,-a\}+B \subseteq \operatorname{Tr}(X, C) \subseteq P+Y$.

Claim. $|B|=|\mathbb{R}|$

To justify the claim, assume that $|B|<|\mathbb{R}|$. Let $S$ be the group generated by the set $B$. Then $|S|<|\mathbb{R}|$. Let $d \in \mathbb{R} \backslash S$. Since $|X+\{a,-a\}+\{0, d\}|=\kappa$, by the definition of $C$, for some $r \in \mathbb{R}$, we have

$$
X+\{a,-a\}+\{0, d\}+r \subseteq C .
$$

This implies that $\{a,-a\}+\{0, d\}+r \subseteq \operatorname{Tr}(X, C)$. Now, by the definition of $B, 0+r$ and $d+r$ belong to the set $B$ and hence $r$ and $d+r$ are in the additive subgroup $S$. Consequently, $d \in S$, which contradicts the choice that $d \in \mathbb{R} \backslash S$. Thus $|B|=|\mathbb{R}|$. To conclude the proof of the lemma, recall that 
(3) $\{a,-a\}+B \subseteq P+Y,|Y|<|\mathbb{R}|$ and $|B|=|\mathbb{R}|$.

For $y$ in $Y$, let $B(y)=\{b \in B: a+b \in P+y\}$. Since $\bigcup_{y \in Y} B(y)=B$, by $(3)$, there exists $y_{1} \in Y$ such that $\left|B\left(y_{1}\right)\right|>|Y|$. Note that $a+B\left(y_{1}\right) \subseteq P+y_{1}$. Now, $-a+B\left(y_{1}\right) \subseteq P+Y$ and $\left|B\left(y_{1}\right)\right|>|Y|$ imply that there exists $y_{2} \in Y$ and an infinite subset $E$ of $B\left(y_{1}\right)$ such that $-a+E \subseteq P+y_{2}$. We have $a+E \subseteq P+y_{1}$ and $-a+E \subseteq P+y_{2}$. For each $e \in E, a+e-y_{1}$ and $-a+e-y_{2}$ are distinct points in $P$ and

$$
\left|\left(a+e-y_{1}\right)-\left(-a+e-y_{2}\right)\right|=\left|2 a-y_{1}+y_{2}\right|
$$

is a constant, which contradicts the property of $P$.

To conclude the proof of the theorem, assume that the conclusion of the theorem is false. Then for some nonempty open interval $I$ and a meager set $F$, we have $I \backslash F \subseteq \bigcup_{i=1}^{n}\left(P_{i}+G_{i}\right)$. Now,

(4) $\mathbb{R} \backslash(F+\mathbb{Q})=(I+\mathbb{Q}) \backslash(F+\mathbb{Q}) \subseteq(I \backslash F)+\mathbb{Q} \subseteq \bigcup_{i=1}^{n}\left(P_{i}+G_{i}+\mathbb{Q}\right)$.

Since $F+\mathbb{Q}$ is meager, it is easy to see that $\mathbb{R} \backslash(F+\mathbb{Q})$ contains a translated copy of every countable subset of $\mathbb{R}$. By applying Lemma $6 n$ times, we obtain that $(\mathbb{R} \backslash(F+\mathbb{Q})) \backslash \bigcup_{i=1}^{n}\left(P_{i}+G_{i}+\mathbb{Q}\right)$ contains a translated copy of every countable subset of $\mathbb{R}$, but according to $(4),(\mathbb{R} \backslash(F+\mathbb{Q})) \backslash \bigcup_{i=1}^{n}\left(P_{i}+G_{i}+\mathbb{Q}\right)$ is an empty set.

\section{References}

[1] K. Ciesielski, L. Larson, Uniformly antisymmetric functions, Real Analysis Exchange, 19 No. 1 (1993-94), 226-235.

[2] K. Ciesielski, Uniformly antisymmetric functions and $K_{5}$, Real Analysis Exchange, 21 No. 1 (1995-96), 147-153.

[3] P. Erdös and S. Kakutani, On non-denumerable graphs, Bulletin of the American Mathematical Society, 49 (1943), 457-461.

[4] P. Erdös, On some properties of Hamel basis, Colloquium Mathematicum, 10 (1963), 267-269.

[5] R. Graham, B. L. Rothschild and J. H. Spencer, Ramsey Theory, Wiley, New York 1980. 
[6] K. Kunen, Partitioning Euclidean space, Mathematical Proceedings of the Cambridge Philosophical Society, 102 No. 3(1987), 379-383.

[7] J. C. Morgan II, On translation invariant families of sets, Colloquium Mathematicum, 34 (1975), 63-68.

[8] H. Miller, On a property of Hamel basis, Bollettino Unione Mathematica Italiana, A(7), 3 (1989), 39-43.

[9] K. Muthuvel, Some topological properties of Hamel basis, Real Analysis Exchange, 20 No. 2 (1994-95), 819-822. 